\title{
Hologram Generation of 3D Objects Using Multiple Orthographic View Images
}

\author{
Min-Su Kim, Ganbat Baasantseren, Nam Kim, and Jae-Hyeung Park* \\ School of Electrical \& Computer Engineering, Chungbuk National University, 410 SungBong-Ro, \\ Heungduk-Gu, Cheongju-Si, Chungbuk, 361-763, Korea
}

(Received October 8, 2008 : revised December 3, 2008 : accepted December 5, 2008)

\begin{abstract}
We propose a new synthesis method for the hologram of 3D objects using incoherent multiple orthographic view images. The 3D objects are captured and their multiple orthographic view images are generated from the captured image. Each orthographic view image is numerically overridden by the plane wave propagating in the direction of the corresponding view angle and integrated to form a point in the hologram plane. By repeating this process for all orthographic view images, we can generate the Fourier hologram of the 3D objects.
\end{abstract}

Keywords: Digital holography, 3D imaging

OCIS codes : (090.0090) Holography; (090.1760) Computer holography; (100.6890) Three-dimensional image processing; (110.0110) Imaging systems

\section{INTRODUCTION}

Holography is an attractive method since it can thoroughly capture and display 3D information about the objects. The generation of the hologram, however, is not easy due to the necessity of a coherent system using a laser [1]. A method synthesizing the hologram with incoherent multi-view images has been reported in order to eliminate the necessity of the coherent image system [2]. However, this method uses perspective projection images as view images, requiring some approximations, to get the desired hologram [3].

In this paper, we propose a new synthesis method for the hologram of 3D objects using incoherent orthographic view images. The 3D objects are captured and their orthographic view images are generated from the captured image [4]. Each orthographic view image is numerically overridden by the plane wave propagating in the direction of the corresponding view angle and integrated into a single complex value, which constitutes one pixel in the synthesized hologram. By repeating this process for all orthographic view images, we can generate the Fourier hologram of the 3D objects. Since the proposed method generates the hologram not from the interference with the reference beam, but from the multiple view images, a coherent system is not required. Moreover, the use of the orthographic projection view image instead of the perspective projection view image results in the exact Fourier hologram without any approximation.

\section{PRINCIPLE}

Angular view images of the 3D objects can be classified into two categories, i.e. perspective image and orthographic image, according to the projection geometry. As shown in Fig. 1, the projection lines converge to one vanishing point in the perspective projection case, while they are all parallel in the orthographic case. Previous research has been based on the perspective projection geography, hence there are limitations on the projection angle $[5,6]$ or the object distance $[3,7]$. The proposed method utilizes the orthographic image instead of the perspective image, leading to the generation of an exact Fourier hologram without any approximation.

Given the projection angles $\theta_{x}$ and $\theta_{y}$, the object point at $(x, y, z)$ is projected to $\left(x_{p}, y_{p}\right)$ in the orthographic projection geometry image following the relation given by

\footnotetext{
*Corresponding author: jh.park@cbnu.ac.kr
} 


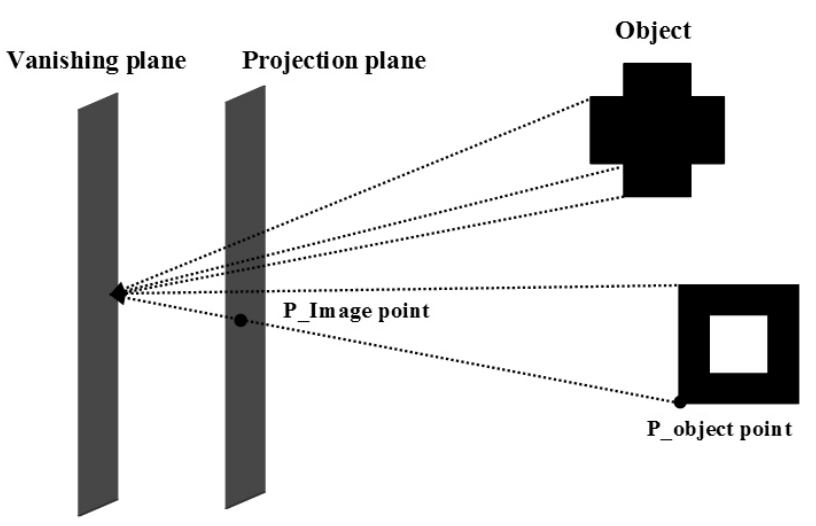

(a)

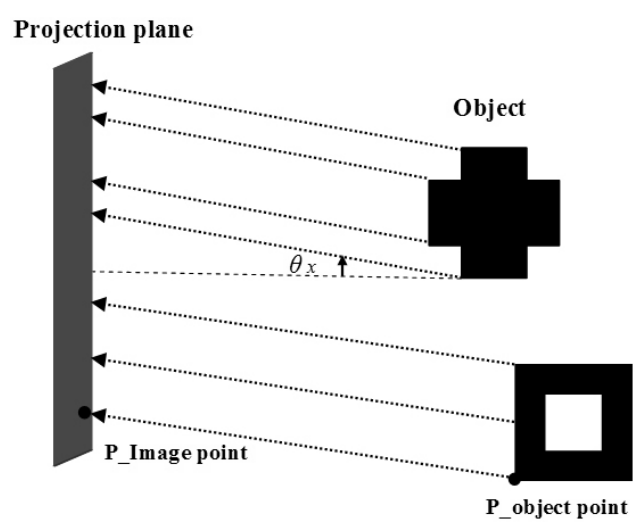

(b)

FIG. 1. Projection geometry (a) Perspective projection method and (b) Orthographic projection method of two 3D objects.

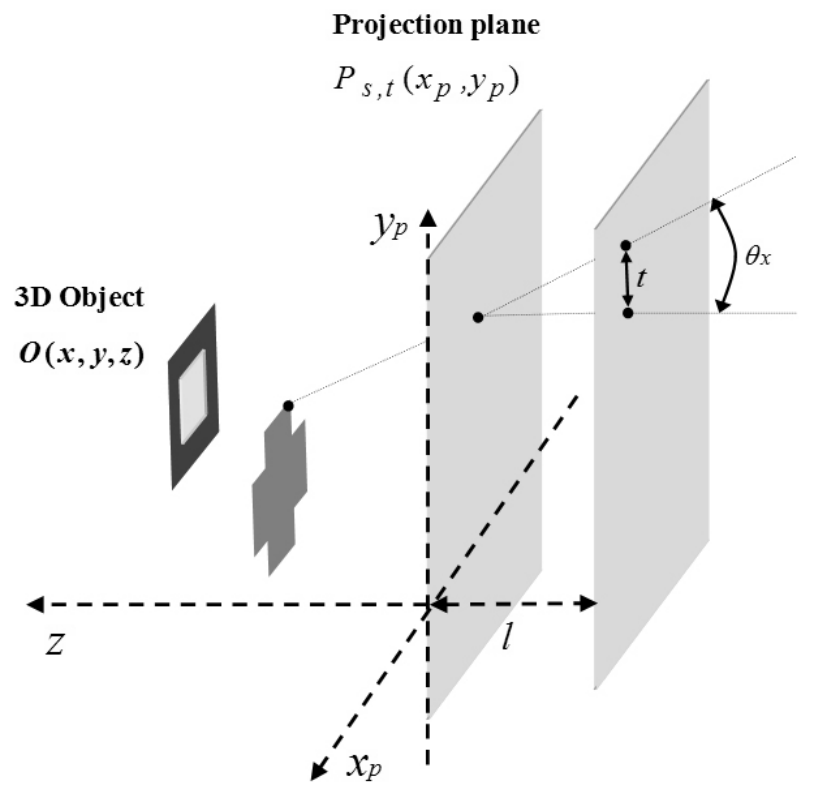

FIG. 2. The coordinate system of objects for orthographic projection.

$$
\begin{aligned}
& x_{p}=x+z \tan \theta_{x}=x+z \frac{s}{l} \\
& y_{p}=y+z \tan \theta_{y}=y+z \frac{t}{l}
\end{aligned}
$$

where $s, t$ and $l$ are defined as shown in Fig. 2. The parameters $s$ and $t$ in Eq. (1) define the projection angles $\theta_{x}$ and $\theta_{y}$ with a fixed value of $l$. In the proposed method, each orthographic view image is multiplied by the plane wave of corresponding projection angle and summed to generate a single complex value in the hologram plane. If we denote the orthographic projection image of the view angle corresponding to $s$ and $t$ as $P_{s, t}\left(x_{p}, y_{p}\right)$, this process can be represented by

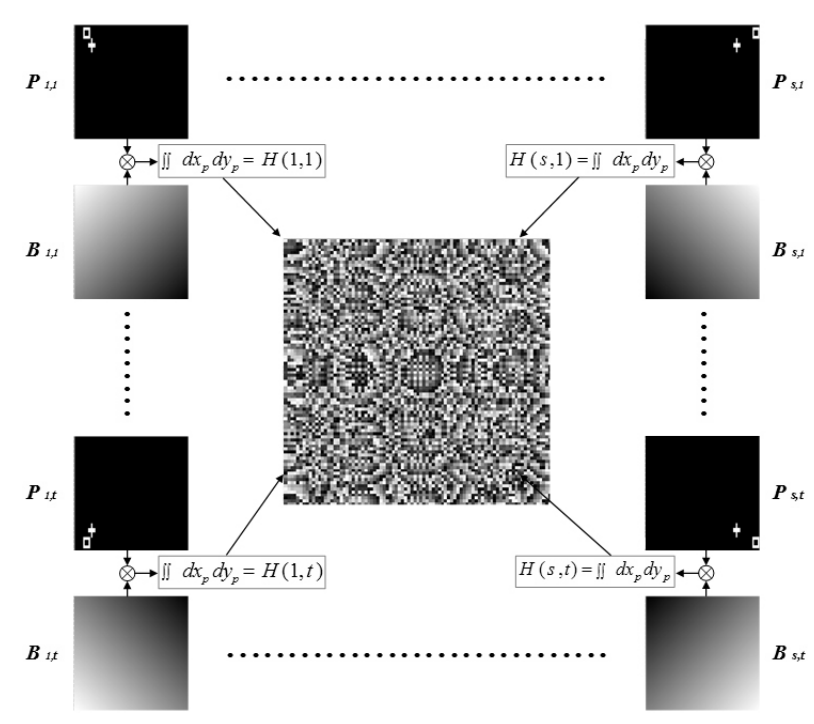

FIG. 3. Schematic diagram of the proposed method.

$H(s, t)=\iint P_{s, t}\left(x_{p}, y_{p}\right) \times \exp \left[-j 2 \pi b\left(x_{p} \cdot s+y_{p} \cdot t\right)\right] d x_{p} d y_{p}$

where $b$ is a constant. Figure 3 shows the proposed method schematically.

Now we show that the proposed method generates the exact Fourier hologram of the $3 \mathrm{D}$ objects. From Eq. (3), the orthographic image point $P_{s, t}\left(x_{p}, y_{p}\right)$ can be replaced by the corresponding object point $\mathrm{O}(x, y, z)=$ $\mathrm{O}\left(x_{p}-z \frac{s}{l}, y_{p}-z \frac{t}{l}, z\right)$, which gives

$H(s, t)=\iint O\left(x_{p}-z \frac{s}{l}, y_{p}-z \frac{t}{l}, z\right) \times \exp \left[-j 2 \pi b\left(x_{p} \cdot s+y_{p} \cdot t\right)\right] d x_{p} d y_{p}$

By substituting $x_{p}-z \frac{s}{l}$ and $y_{p}-z \frac{t}{l}$ with $x$ and $y$ 


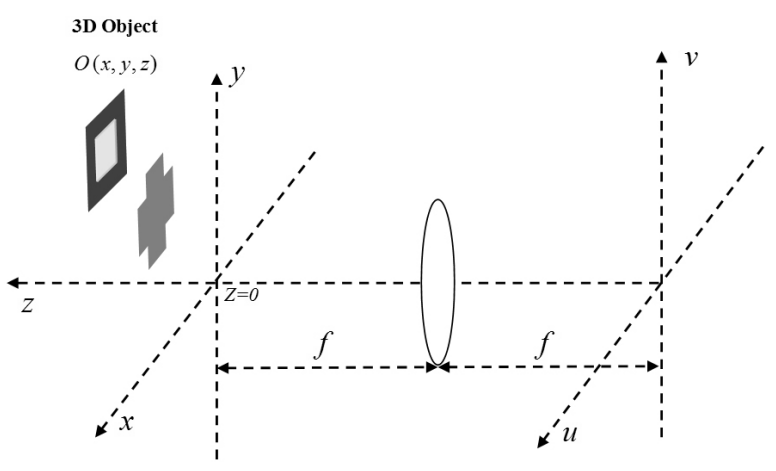

FIG. 4. Geometry of Fourier hologram of 3D objects.

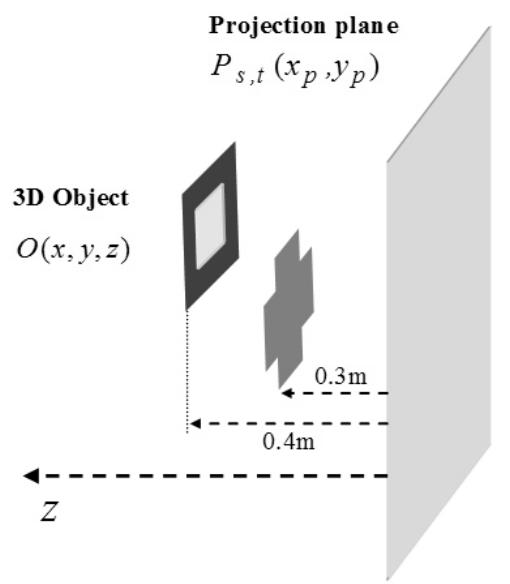

FIG. 5. Geometry of two plane objects used in the simulation.

in Eq. (3), Eq. (3) becomes

$H(s, t)=\iiint O(x, y, z) \times \exp \left[-j 2 \pi b\left\{(x \cdot s+y \cdot t)+\frac{z}{l}\left(s^{2}+t^{2}\right)\right\}\right] d x d y d z$

Here, $(s, t)$ are discrete values defining the view angle of the orthographic view image. If we denote the scaled coordinates on the hologram plane as $(u, v)$, finally we get

$H(u, v)=\iiint O(x, y, z) \times \exp \left[-j \frac{2 \pi b}{M}\left\{x \cdot u+y \cdot v+\frac{z}{l M}\left(u^{2}+v^{2}\right)\right\}\right] d x d y d z$

where $M=\frac{u}{s}=\frac{\nu}{t}$ is a coordinate magnification factor.

In geometry shown in Fig. 4, the complex field $\mathrm{g}(u, v)$ of the 3D object at the Fourier plane of a Fourier transform lens of a focal length $f$ is given by [8]

$g(u, v)=A \iiint O(x, y, z) \times \exp \left[-j \frac{2 \pi}{\lambda f}\left\{x \cdot u+y \cdot v-\frac{z}{2 f}\left(u^{2}+v^{2}\right)\right\}\right] d x d y d z$

where $A$ is a constant. From Eqs. (5) and (6), we can see that the hologram generated by the proposed method has the same form as the Fourier hologram, provided that

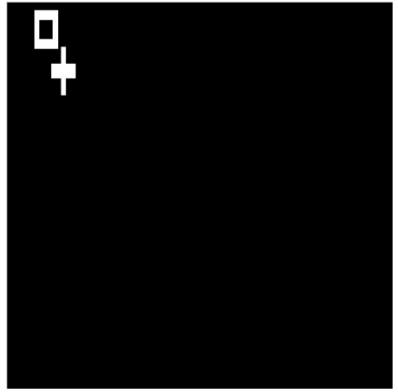

(a)

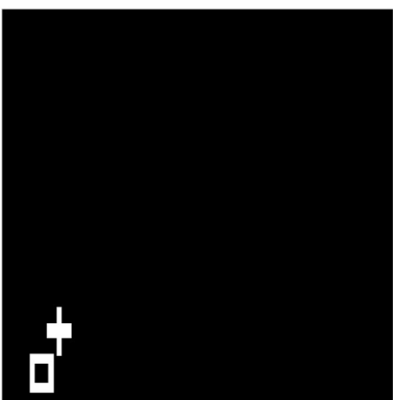

(c)

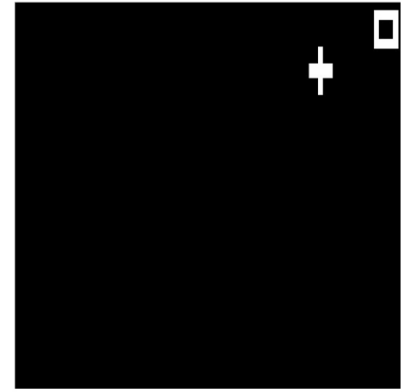

(b)

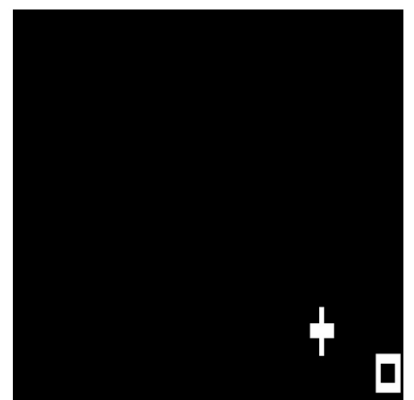

(d)
FIG. 6. Orthographic view images for two plane objects (a) left top, (b) right top, (c) left bottom and (d) right bottom.

$$
M=\frac{-2 f}{l}, \quad b=\frac{-2}{\lambda l}
$$

Consequently, the proposed method generates the exact Fourier hologram without any approximations.

\section{SIMULATION AND EXPERIMENTAL RESULTS}

We verified the proposed method by simulation and experiment. In simulation, two plane objects located at $0.3 \mathrm{~m}$ and $0.4 \mathrm{~m}$ from the projection plane are used as shown in Fig. 5. The range of the horizontal angle $\theta_{x}$ and vertical angle $\theta_{y}$ in simulation are -5 degree to 5 degree with 87 steps for each direction. A few examples of the orthographic projection images are shown in Fig. 5. We can see that the relative position of two objects varies according to the view angle. Note that the rectangular object has larger disparity than the cross object. In contrast to the perspective projection case where the disparity is inversely proportional to the object depth, in orthographic projection geometry the disparity is proportional to the object depth as can be easily verified from Eq. (1). Therefore larger disparity of the rectangular object in Fig. 6 is a direct consequence of the orthographic projection. 


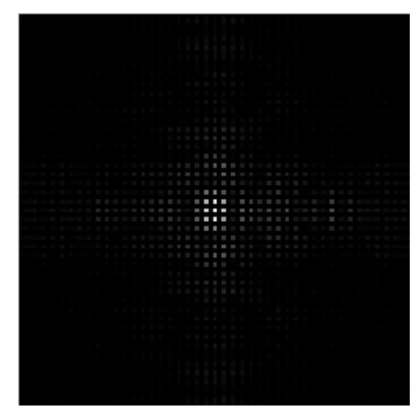

(a)

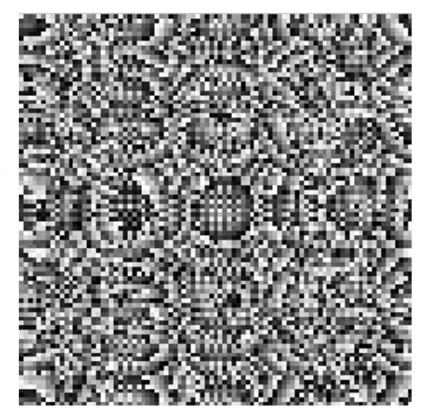

(b)
FIG. 7. Generated Fourier hologram of the two plane images (a) The magnitude and (b) the phase angle $(87 \times 87$ pixels).

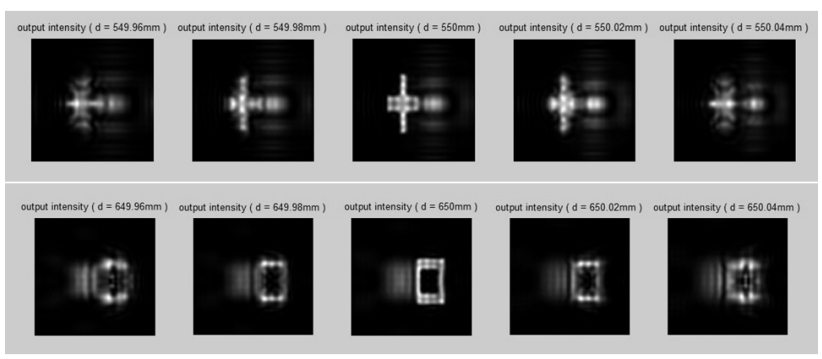

FIG. 8. Simulation result of the two plane objects: reconstruction at different depths.

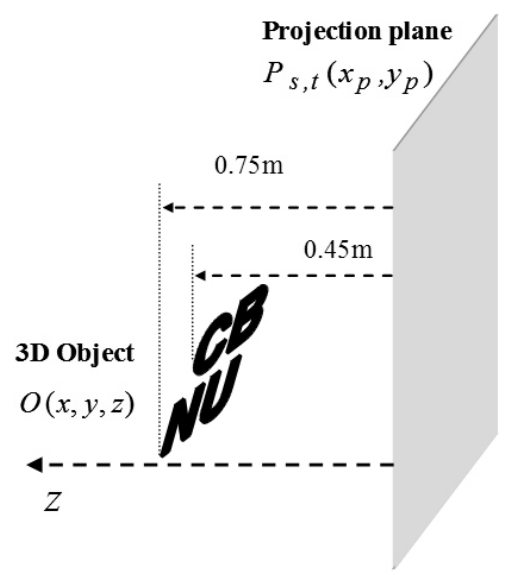

FIG. 9. Geometry of the tilted plane object with continuous depth.

Using $87 \times 87$ orthographic projection images, the Fourier hologram is synthesized by Eq. (2). In the synthesis, the focal length of the lens $f$ and the wavelength $\lambda$ were assumed to be $0.25 \mathrm{~m}$ and $532 \mathrm{~nm}$, respectively. The complex field of the synthesized hologram is shown in Fig. 7. Since $87 \times 87$ orthographic projection images are used, the resultant hologram has $87 \times 87$ pixels. In order to verify the synthesized hologram, we reconstruct the object space by Fresnel propagating the synthesized hologram using

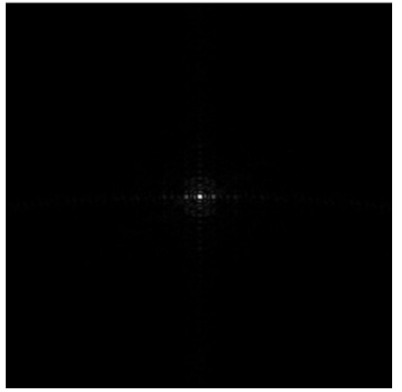

(a)

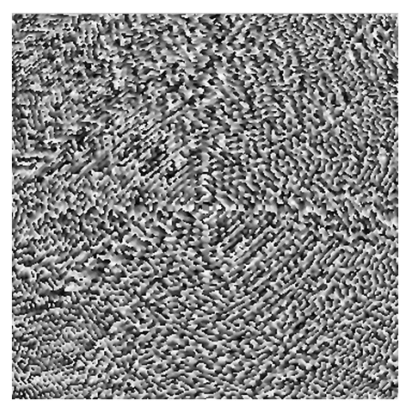

(b)
FIG. 10. Generated Fourier hologram of the tilted plane object with continuous depth (a) The magnitude and (b) the phase angle $(361 \times 361$ pixels $)$.

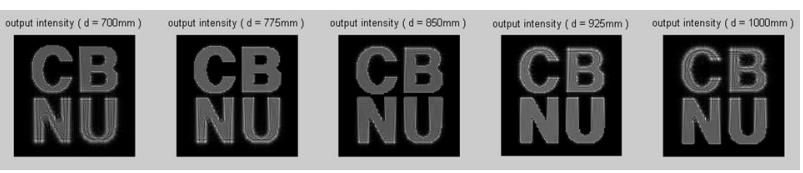

FIG. 11. Simulation result of the tilted plane object: reconstruction at different depths.

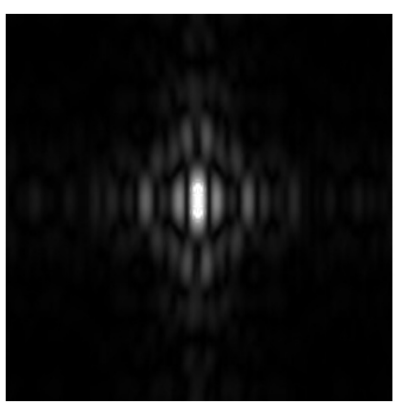

FIG. 12. Enlarged part $(200 \times 200$ pixels of $767 \times 767$ pixels) of the modified hologram $H^{\prime}(u, \nu)$.

$U_{d}(x, y)=A \iint \exp \left[j \frac{k}{2 f}\left(1-\frac{d}{f}\right)\left(u^{2}+v^{2}\right)\right] \times H(u, v) \exp \left[-j \frac{2 \pi}{\lambda f}(u x+v y)\right] d u d v$

where $d$ is the propagation distance after the Fourier transform lens [9]. The reconstruction result is shown in Fig. 8.

We can see that the cross object is reconstructed at $\mathrm{d}=500 \mathrm{~mm}=f+300 \mathrm{~mm}$ and the rectangular object is reconstructed at $d=650 \mathrm{~mm}=f+400 \mathrm{~mm}$ as expected. Therefore we can confirm that the synthesized hologram correctly contains the 3D information of the object.

In addition, we conducted a simulation for the $3 \mathrm{D}$ object having continuous depth. A plane object tilted in $y-z$ plane as shown in Fig. 9 is used in the simulation. The depth is continuous from $0.45 \mathrm{~m}$ for the uppermost part to $0.75 \mathrm{~m}$ for the downmost part. Figure 10 shows the synthesized hologram. The reconstruction result at different depths is shown in Fig. 11. We can recognize that the $3 \mathrm{D}$ object is reconstructed in the range spanning 


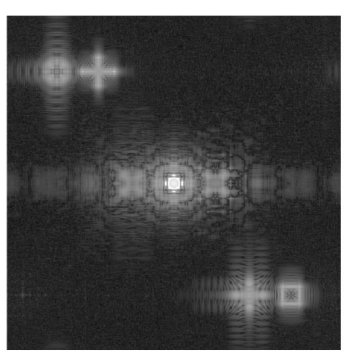

(a)

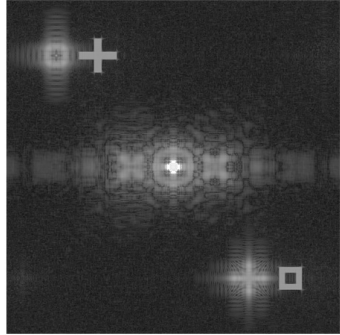

(b)

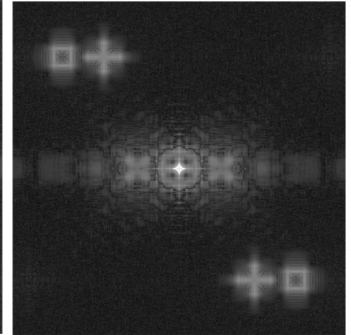

(c)

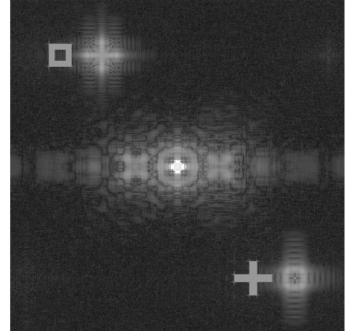

(d)

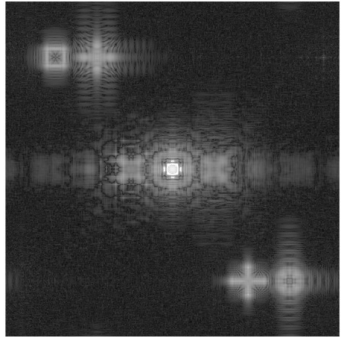

(e)

FIG. 13. Simulation results: reconstruction using modified hologram $H^{\prime}(u, \nu)$ at (a) $d=658 \mathrm{~mm}$, (b) $d=688 \mathrm{~mm},(\mathrm{c})$ $d=718 \mathrm{~mm}$, (d) $d=748 \mathrm{~mm}$, and (e) $d=778 \mathrm{~mm}$.

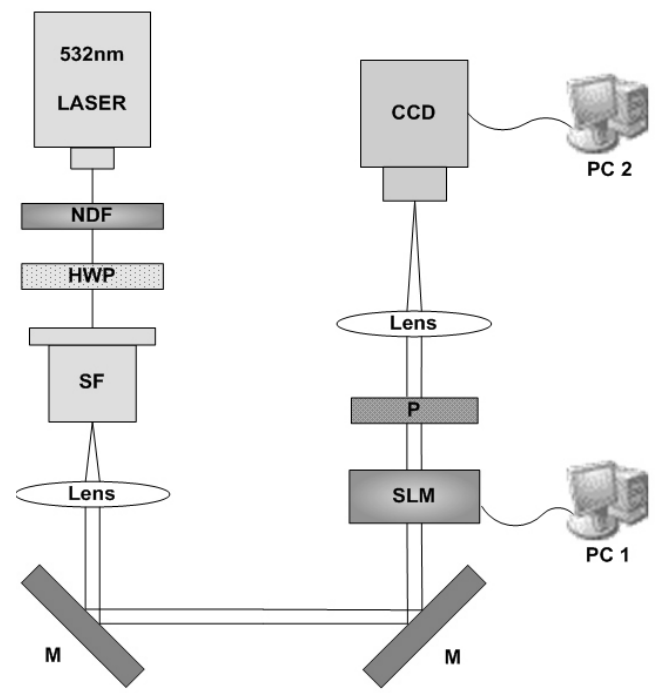

\author{
NDF : Neutral Density Filter \\ HWP : Half wave plate \\ SF : Spatial filter \\ M : Mirror \\ $\mathrm{P}$ : Polarizer \\ BS : Beam splitter \\ SLM : Spatial light modulator \\ PC 1 : Personal computer 1 for SLM \\ PC 2 : Personal computer 2 for CCD
}

FIG. 14. Optical experiment setup for reconstruction of hologram using the amplitude modulation type SLM.
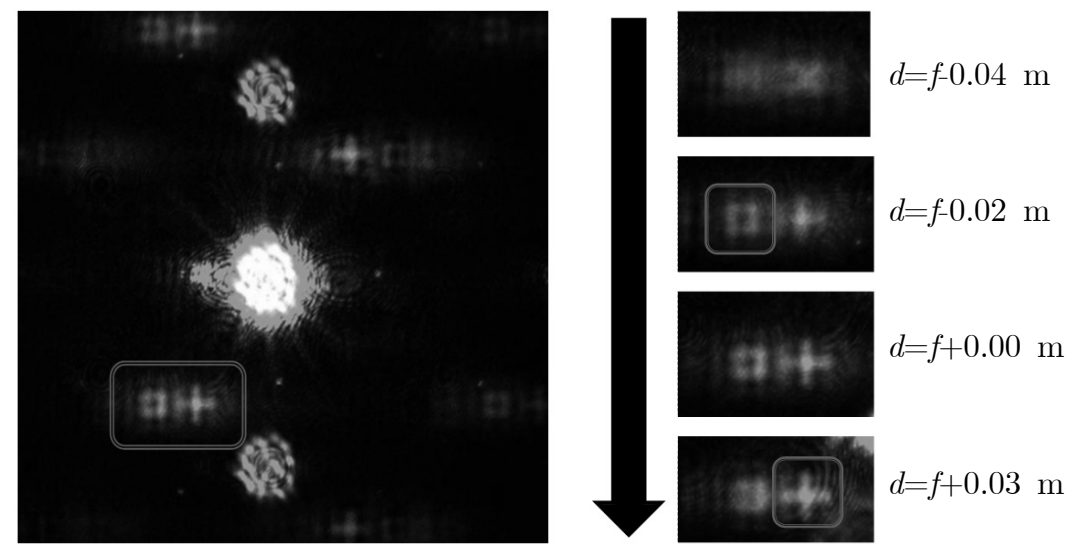

FIG. 15. Experimental result: reconstruction at different depths.

from $d=700 \mathrm{~mm}=f+450 \mathrm{~mm}$ to $d=1000 \mathrm{~mm}=f+750 \mathrm{~mm}$ with only the corresponding part focused.

We performed an experiment as well. In the experiment, we optically reconstruct a 3D scene using the synthesized hologram. The hologram is generated by the proposed method assuming two objects are located at $f-0.02 \mathrm{~m}$ and $f+0.03 \mathrm{~m}$, respectively and the focal length is $0.25 \mathrm{~m}$. $767 \times 767$ orthographic images were used to generate the hologram. Since the only SLM which was available at the time of the experiment is amplitude type SLM, we could not get the sharp 3D image as in the simulation. Note that the simulation results shown in Fig. 8 and Fig. 11 were obtained using both of the amplitude and phase of the synthesized hologram. In order to improve the diffraction efficiency, we generate new hologram $H^{\prime}(u, \nu)$ which is given by $[10]$ 


$$
H^{\prime}(u, v)=\frac{1}{2}\left[\left|H^{\prime \prime}(u, v)\right|+\operatorname{Re}\left\{H^{\prime \prime}(u, v)\right\}\right]
$$

where $H^{\prime \prime}(u, \nu)$ is the normalized version of original hologram $H(u, \nu)$. Figure 12 shows the enlarged central part of $H^{\prime}(u, \nu)$. Note that $H^{\prime}(u, \nu)$ is a purely real positive function which can be realized by the amplitude SLM. Figure 13 is the simulation result using $H^{\prime}(u, \nu)$ instead of $H(u, \nu)$. We can see that the zero-th order and conjugate images appear due to the amplitude only modulation. However still we can observe that the two objects are reconstructed at the correct depth plane.

Using $H^{\prime}(u, \nu)$ and amplitude SLM, we performed optical reconstruction. The SLM used in the experiment has $1024 \times 768$ pixels with $36 \times 36 \mu \mathrm{m}$ pixel pitch. The light source is a Nd-YAG laser with $\lambda=532 \mathrm{~nm}$. The experimental setup is shown in Fig. 14. The optical reconstruction result is shown in Fig. 15. Although it is not as sharp as the simulation result, we can observe that the two objects are focused at different depth planes. Therefore the validity of the proposed method is verified, confirming its feasibility.

\section{DISCUSSION AND CONCLUSION}

In this paper, we proposed a new synthesis method for the Fourier hologram of the 3D objects using orthographic view images in an incoherent system. An orthographic view image is multiplied by the corresponding plane wave and integrated into a single pixel of the hologram plane. By repeating this process for numerous orthographic view images, Fourier hologram of the 3D objects can be synthesized. The proposed method is simple in structure because it is an incoherent process. In contrast to the previous research, the proposed method uses the orthographic image, which eliminates any need for limitations on the maximum view angle or the object distance. We verified the proposed method by simulation and experiment, and the results confirm the feasibility of the proposed method.

\section{ACKNOWLEDGMENT}

"This work was supported by the grant of the Korean Ministry of Education, Science and Technology" (The Regional Core Research Program/Chungbuk BIT ResearchOriented University Consortium)

\section{REFERENCES}

[1] K.-Y. Lee, S.-H. Jeung, D.-D. Do, N. Kim, J.-W. An, "Holographic Demultiplexer with Low Polarization Dependence Loss using Photopolymer Diffraction Gratings," J. Opt. Soc. Korea, vol. 11, no. 2, pp. 51-54, 2007.

[2] Y. Li, D. Abookasis, and J. Rosen, "Computer-generated holograms of three-dimensional reali-stic objects recorded without wave interference," Appl. Opt., vol. 40, no. 17, pp. 2864-2870, 2001.

[3] B. Katz, N. T. Shaked, and J. Rosen, "Synthesizing computer generated holograms with reduced number of perspective projections," Opt. Exp., vol. 15, no. 20, pp. 13250-13255, 2007.

[4] J.-H. Park, G. Baasantseren, N. Kim, G. Park, J. Kang, and B. Lee, "View image generation in perspective and orthographic projection geometry based on integral imaging," Opt. Exp., vol. 16, no. 12, pp. 8800-8813, 2008.

[5] Y. Sando, M. Itoh, and T. Yatagai, "Holographic threedimensional display synthesized from three-dimensional Fourier spectra of real existing objects," Opt. Lett., vol. 28, no. 24, pp. 2518-2520, 2003.

[6] D. Abookasis and J. Rosen, "Computer-generated holograms of three-dimensional objects synthesized from their multiple angular viewpoints," J. Opt. Soc. Am. A, vol. 20, no. 8, pp. 1537-1545, 2003.

[7] N. T. Shaked, J. Rosen, and A. Stern, "Integral holography: white-light single-shot hologram acquisition," Opt. Exp., vol. 15, no. 9, pp. 5754-5760, 2007.

[8] J. W. Goodman, Introduction to Fourier Optics, 2nd ed. (McGraw-Hill, New York, 1996), pp. 104-105.

[9] D.-H. Shin and E.-S. Kim, "Computational Integral Imaging Reconstruction of 3D Object Using a Depth Conversion Technique," J. Opt. Soc. Korea, vol. 12, no. 3, pp. 131-135, 2008.

[10] J. J. Burch, "A computer algorithm for the synthesis of spatial frequency filters," Proc. IEEE, vol. 55, no. 4, pp. 599-600, 1967. 